\title{
Pleural mechanics and the pathophysiology of air leaks
}

Video clip is available online.

An understanding of pleural physiology has practical implications for the management of the perioperative thoracic surgical patients as well as patients with spontaneous or traumatic disruption of the pleura. Effective management of the pleural space can optimize lung volumes while maintaining effective ventilation; ineffective management can result in low lung volumes and the potentially lifethreatening compromise of gas exchange.

Pleural pressures reflect the pressure dynamics of the chest. Although there is some variation with posture, pleural pressures are a common intrathoracic pressure; that is, the pressure on the outside of the lung. ${ }^{1}$ The difference between pleural pressure and airway pressure is the transpulmonary pressure. ${ }^{2,3}$ In the normal respiratory system, during quiet breathing, the transpulmonary pressure causing tidal ventilation is the result of cyclic changes in pleural pressure. In contrast, during mechanical ventilation, the transpulmonary pressure causing tidal ventilation is primarily the cyclic changes in airway pressure.

The recent clinical interest in measuring transpulmonary pressure reflects the effect of transpulmonary pressure on short- as well as long-term outcomes of patients receiving mechanical ventilation. ${ }^{4}$ In spontaneously or mechanically ventilating patients, the determination of transpulmonary pressure requires a measure of intrathoracic pressure; typically, this measurement can be estimated using an esophageal balloon catheter positioned in the distal third of the esophagus (Figure 1). The airway pressure is measured at the mouth or endotracheal tube. Additional measures that are useful in characterizing intrathoracic dynamics are lung volumes and air flow. Air flow can be measured using a pneumotachometer. Lung volume changes can be quantified as integrated air flow, and absolute lung volumes can be measured in a plethysmograph (body box). ageal balloon.

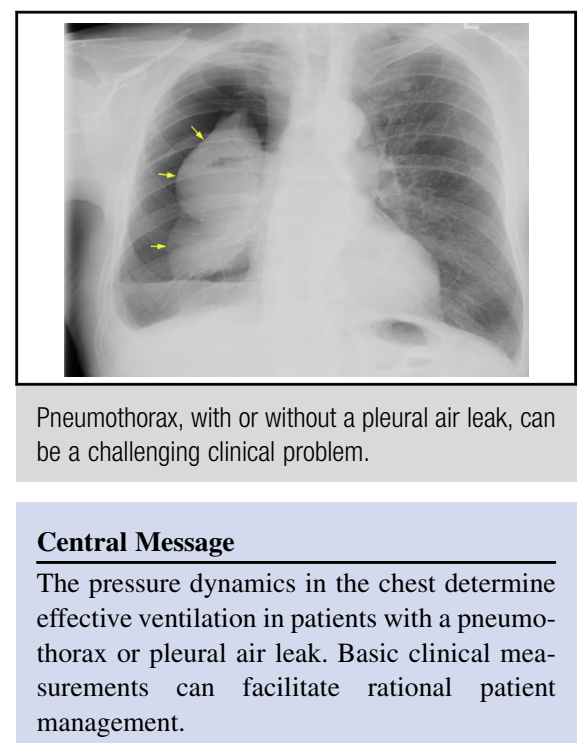

\section{Perspective}

The pressure dynamics in the chest determine effective ventilation in patients with a pneumothorax or pleural air leak. Basic clinical measurements can facilitate rational patient management.

See Editorial Commentary page 2190.

To measure static transpulmonary pressure in the spontaneously breathing patient, pressures are assessed in the absence of air flow. After placement of an esophageal balloon, the patient is asked to take a very deep breath. As the patient expires through the pneumotachometeroften with a side port pressure sensor-the expiratory flow is intermittently occluded. During these static (or quasistatic) maneuvers, the pressure difference between the mouth $\left(\mathrm{P}_{\mathrm{Aw}}\right)$ and esophageal balloon pressure $\left(\mathrm{P}_{\mathrm{Es}}\right)$ provides a measure of transpulmonary pressure (Figure 1). Similar measurements of transpulmonary pressure can be obtained in patients receiving mechanical ventilation using an esoph-

\section{PLEURAL INJURY}

The importance of pleural mechanics is highlighted by pneumothoraces and pleural air leaks. Pneumothorax, a collection of air in the pleural space, can be either stable or unstable. A stable pneumothorax is an unchanging volume of air in the chest that might compromise pleural pressures and lung volumes. In contrast, an unstable 


\section{Abbreviations and Acronyms \\ $\mathrm{P}_{\mathrm{Aw}}=$ airway pressure \\ $\mathrm{P}_{\mathrm{Es}}=$ esophageal balloon pressure \\ $\mathrm{PV}=$ pressure-volume}

pneumothorax is characterized by an expanding volume of air in the pleural space. A common cause of an unstable pneumothorax is a pleural air leak. Air leaks are most frequently associated with pulmonary surgery, ${ }^{5,6}$ but can also be the result of trauma or the spontaneous rupture of pleural blebs. Undrained, an expanding accumulation of air in the pleural space is associated with a progressive loss of lung volume and increasing pleural pressure.

During normal spontaneous breathing (Figure 2), air flow and lung volumes reflect the cyclic variation of pleural pressure. The transition between inspiration and expiration reflects the greatest lung volume, at the point of 0 air flow, just after the lowest pleural pressures (Figure 2, A). Note that the integrated air flow curves during inspiration and expiration are equal. In contrast to normal ventilation, a patient with a stable $20 \%$ pneumothorax will show decreased absolute lung volumes, diminished air flow, and elevated pleural pressures (Figure 2, B). Tidal volumes might be low because of elevated lung resistance and elastance. In patients with an ongoing pleural air leak, a tube in the pleural space provides an opportunity to remove pleural air and reestablish an optimal pleural pressure. Immediately after placement of the pleural tube, positive pleural pressure is reflected by a rush of air out of the chest-analogous to the rush of fluid from a pleural effusion. ${ }^{7}$ Drainage of the pneumothorax is facilitated by applying negative suction through the tube. Because normal pleural pressures are in the range of 0 to $-10 \mathrm{~cm} \mathrm{H}_{2} \mathrm{O}$, a common, but not univer$\mathrm{sal}^{8}{ }^{8}$ clinical practice is to empirically apply $-20 \mathrm{~cm} \mathrm{H}_{2} \mathrm{O}$ external suction to the chest tube. ${ }^{9}$ For most small air leaks, this practice insures that the amount of air evacuated equals or exceeds the amount of air entering the pleural space during breathing (Figure 2, C).

During mechanical ventilation, air flow and lung volumes also reflect the cyclic variation of transpulmonary pressures. Although positive airway pressure as well as negative pleural pressures can contribute to transpulmonary pressure, it is the positive airway pressure that is dominant in patients requiring mechanical ventilation (Figure 3,A). Analogous to spontaneous ventilation, a stable pneumothorax will reduce tidal volumes and air flow (Figure 3, B). An air leak in the setting of mechanical ventilation is inherently unstable and will commonly result in an expanding pleural air collection and tension pneumothorax (not shown). ${ }^{10}$ When the air leak is successfully treated by a chest drain on suction, pleural pressures and tidal volumes return to normal; however, air flow (tidal volume + leak volume) is increased and biased toward inspiration (Figure 3, C).
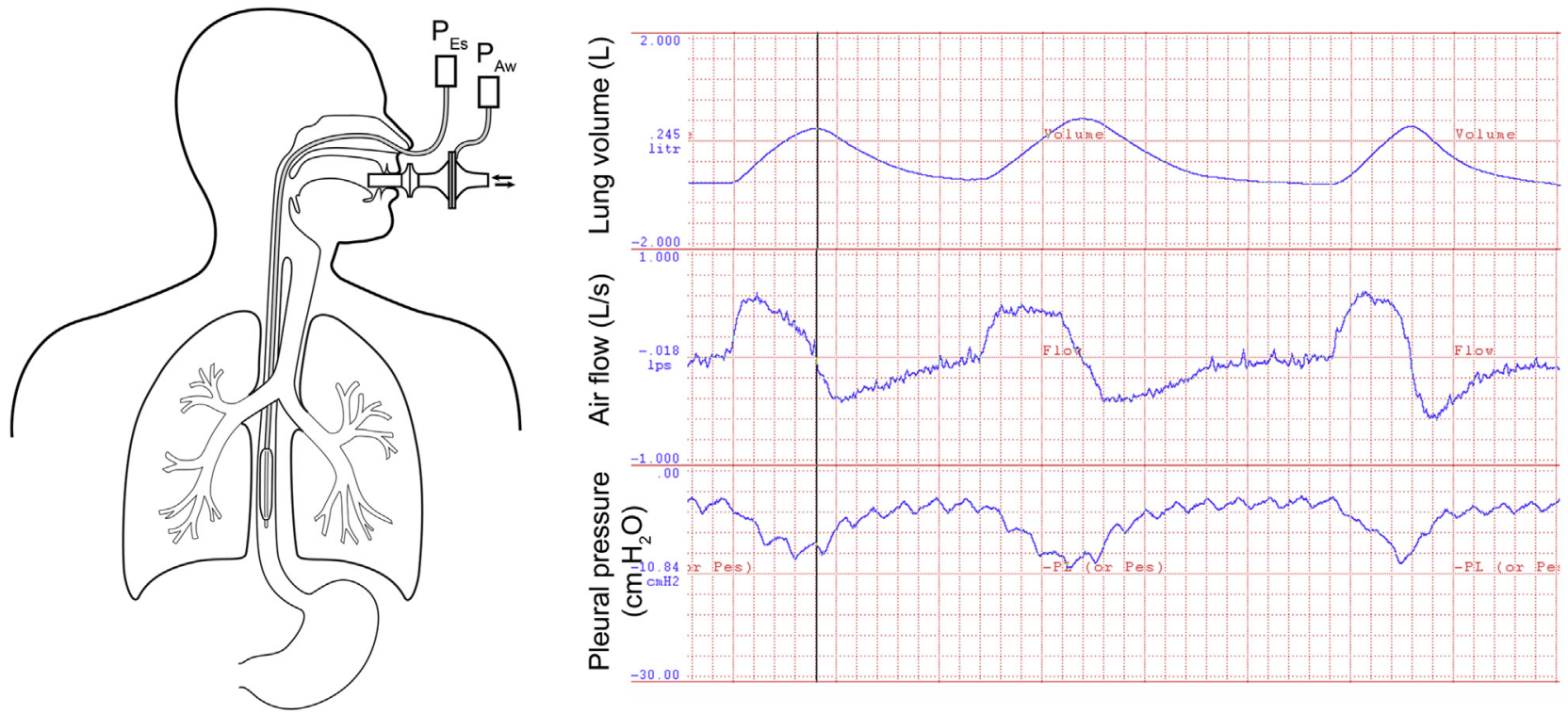

FIGURE 1. In the spontaneously breathing patient, the difference between airway pressure and pleural pressure (transpulmonary pressure) determines air flow and lung volumes. Pleural pressure is estimated by measuring esophageal pressure $\left(P_{E s}\right)$ with an esophageal balloon in the distal third of the esophagus; airway pressure $\left(P_{A w}\right)$ and air flow are measured at the mouth with a pressure sensor and pneumotachometer. Transpulmonary pressure is the difference between $\mathrm{P}_{\mathrm{Aw}}$ and $\mathrm{P}_{\mathrm{Es}}$. In the normal person, the transpulmonary pressure varies with inspiration and expiration; increasing transpulmonary pressure results in increased lung volumes and inspiratory air flow. The cursor shows the transition from inspiratory to expiration at end-inspiratory lung volume (see text). 
Spontaneous breathing
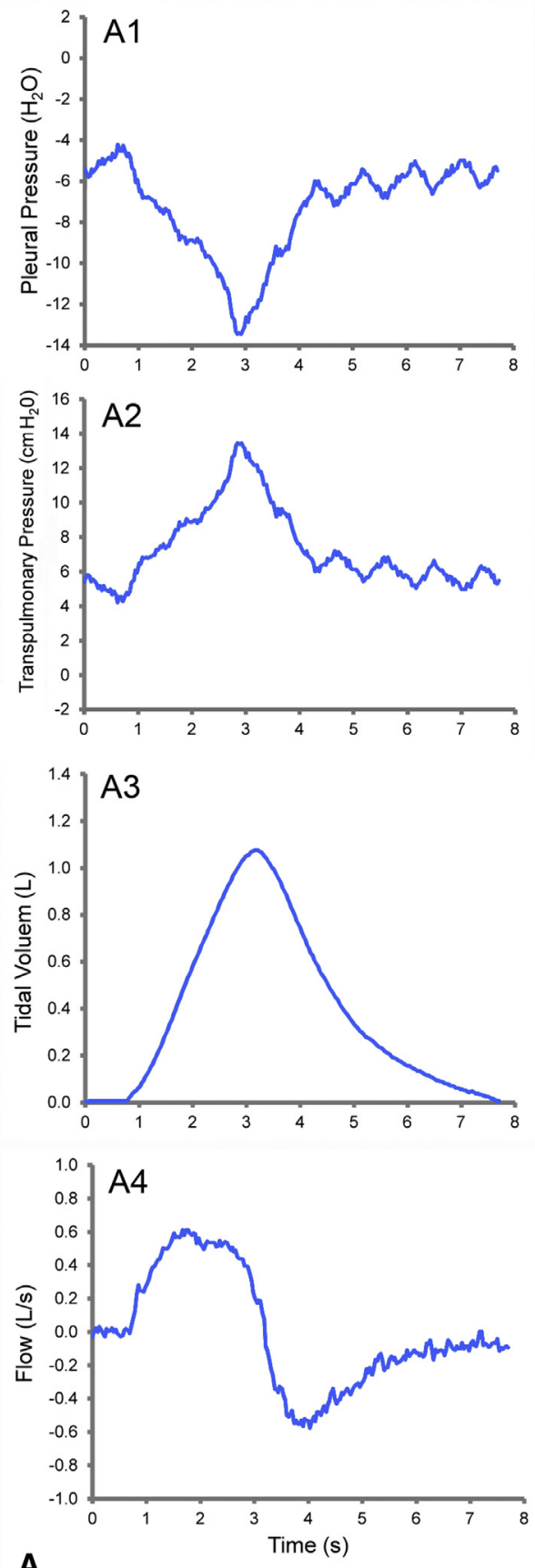

A
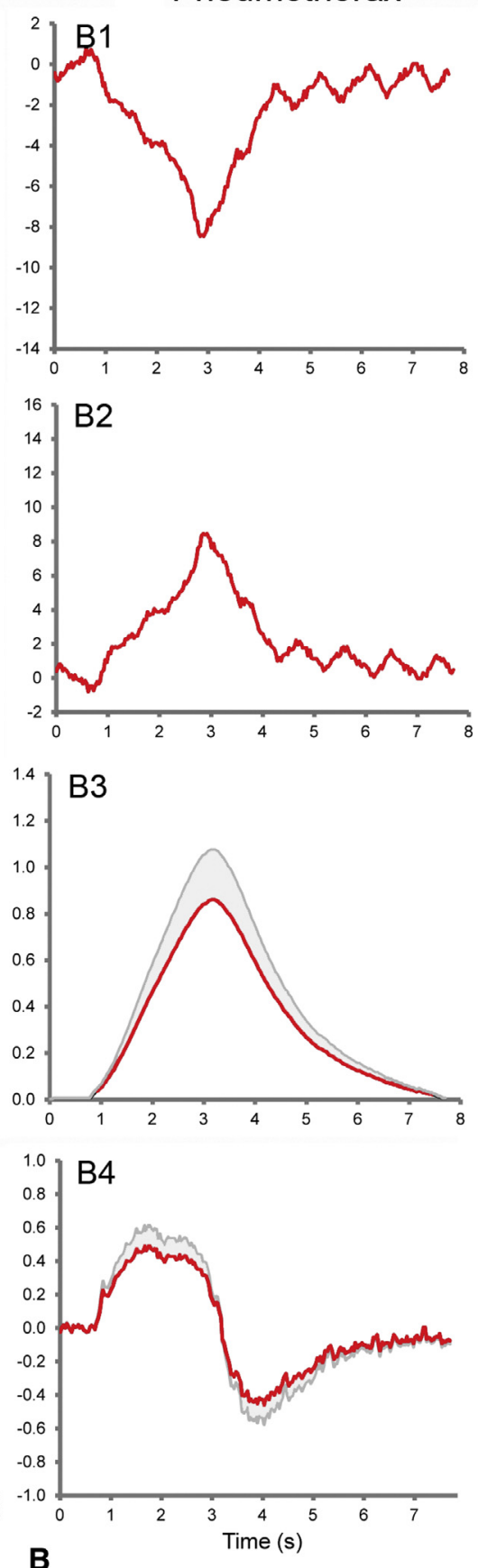

Chest tube
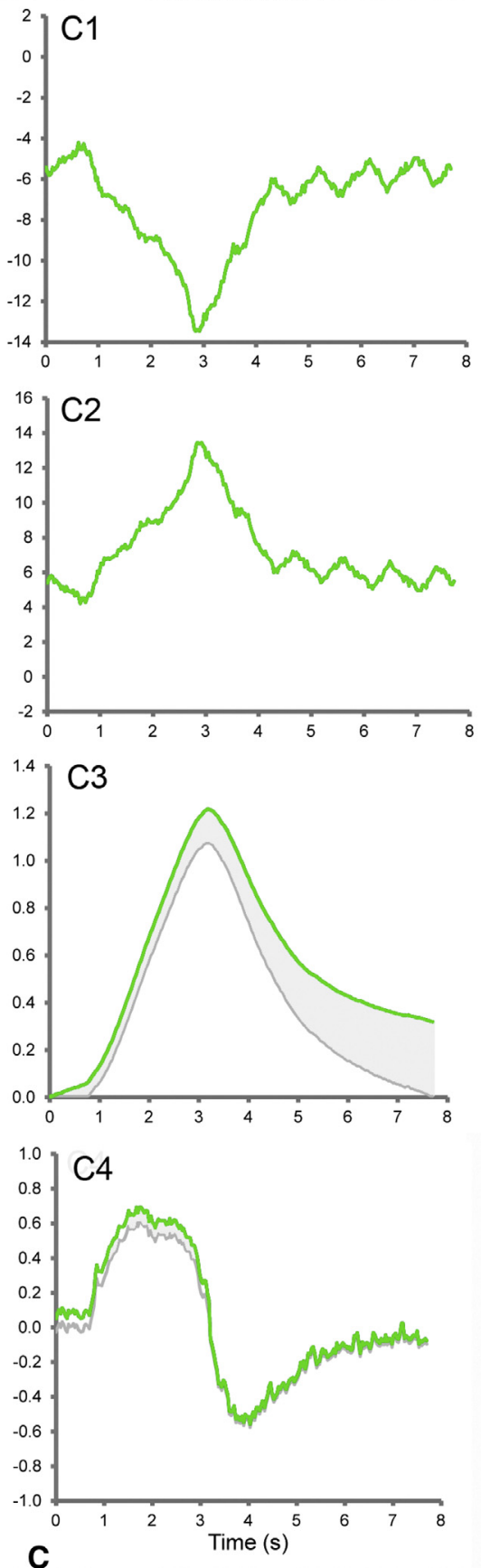

FIGURE 2. Pleural mechanics during spontaneous breathing. Spontaneous breathing under normal conditions (A), spontaneous breathing with a stable pneumothorax (B), and spontaneous breathing with an air leak controlled by a chest tube (C) are shown. Pleural pressures, normally negative (A1), rise with a stable pneumothorax (B1). Pleural pressures return to normal with a chest tube even in the setting of an ongoing air leak (C1). Transpulmonary pressures, reflecting the difference between mouth $\left(0 \mathrm{~cm} \mathrm{H}_{2} \mathrm{O}\right)$ and pleural pressures, mirror the pleural pressures (A2, B2, C2). Because of the elevated pleural pressures, absolute lung volumes are lower with an undrained pneumothorax, and tidal volumes might also be lower because of increase in lung resistance and elastance (B3, gray). In contrast, inspiratory tidal volumes are higher and expiratory tidal volumes are lower than normal in patients with an ongoing air leak (C3, gray). Tidal volumes do reach baseline because of the leak volume out of the chest tube. Similarly, tidal air flow with spontaneous breathing (A4) might be lower with a stable pneumothorax (B4), but higher (with an inspiratory bias) with an air leak controlled by a chest tube (C4). 



B

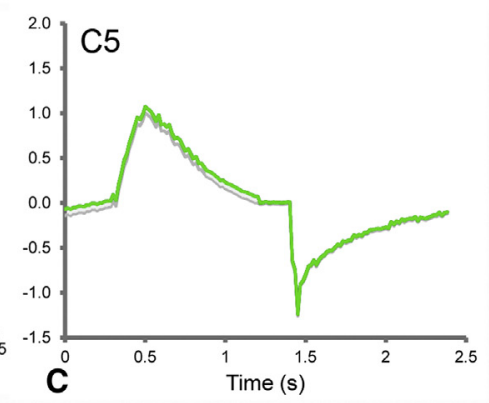

FIGURE 3. Pleural mechanics during mechanical ventilation. Mechanical ventilation without pneumothorax or air leak (A) is compared with mechanical ventilation with a stable pneumothorax (B) and mechanical ventilation with an air leak controlled by a chest tube (C). With pressure-cycled ventilation, the airway pressures will be similar in the 3 conditions. Pleural pressures will rise with a stable pneumothorax (B2). Pleural pressures return to normal with a chest tube despite an ongoing air leak (C2). Transpulmonary pressures, reflecting the difference between airway and pleural pressures, are significantly lower with a pneumothorax (B3). Because of the compromised pleural pressures, absolute lung volumes and tidal volumes are lower with an undrained pneumothorax (B4, gray). In contrast, inspiratory tidal volumes are higher than normal in patients with a controlled air leak and do not return to baseline because of leak volume loss (C4, gray). Similarly, inspiratory air flow is higher and expiratory airflow is lower with an air leak controlled by a chest tube (C5, gray). 


\section{Pleural Mechanics and the Pathophysiology of Air Leaks}

\section{PLEURAL PRESSURES}

\section{Normal Respiratory Variation}

The clinical chest tube and traditional pleural drainage system provide an opportunity to observe pleural pressure variations and note physiologic changes in the respiratory system (Video 1). The fluid column associated with pleural tubes normally varies with ventilation. In patients who are breathing spontaneously, the fluid column rises with inspiration and falls with expiration. A loss of this normal "respiratory variation" likely means that the tube is no longer exposed to intrathoracic pressures. The loss of respiratory variation suggests the importance of looking for external factors (eg, kinked tube) that might contribute to the apparent blunting of pleural pressure swings. In most cases, the chest tube is physically excluded from global pleural pressures by the anatomic location of the tube, normal pleural healing, or pathologic changes (eg, blood clots).

\section{Expiratory Air Leak}

If the pleural tube is adequately draining the pleural space, and there is no air accumulation, the system will rapidly reach equilibrium. With the application of fixed suction (eg, $-20 \mathrm{~cm} \mathrm{H}_{2} \mathrm{O}$ ) to the chest tube, the local pleural pressure might be slightly more negative than normal. The common observation of a phasic or "expiratory" air leak in a pleural drainage system reflects the relative dynamics of pleural pressure and chest tube suction. If inspiratory pleural pressures fall below the tube suction pressure, air will accumulate in the pleural space during this phase of the respiratory cycle. On expiration, pleural pressures rise above suction pressure and the air accumulated on inspiration will be evacuated by the chest tube. Another method for creating a gradient between pleural pressure and tube pressure is coughing. Coughing transiently increases pleural pressure and facilitates the evacuation of pleural air.

\section{Continuous Air Leaks}

In patients with very large air leaks - that is, air leaks approaching the volume of tidal inspiration-evacuation of pleural air will influence not only pleural mechanics, but also gas exchange. The effect of a large air leak on $\mathrm{CO}_{2}$ reflects the diminished partial pressure of $\mathrm{CO}_{2}$ in the peripheral airspaces. An evacuated air leak removes $\mathrm{CO}_{2}$, decreases the concentration of $\mathrm{CO}_{2}$ in the alveoli, and enhances the diffusion gradient from the blood into the alveoli. ${ }^{11}$ The result can be a marked decline in blood $\mathrm{CO}_{2}$ and a significant respiratory alkalosis. ${ }^{12}$

The effect of a large air leak on oxygenation can be twofold. If the large air leak is not controlled by the chest tubes, the accumulating pleural air can reduce lung volumes and compromise oxygenation. Alternatively, if the chest tube suction influences central airway pressures - that is, through a central bronchopleural fistula- then the inability of the suction to control the pleural pressure might decrease transpulmonary pressures and lung volumes. The loss of transpulmonary pressure due to a large air leak has been aptly called a "steal" phenomenon. In these cases, the smaller lung volumes decrease the efficiency of gas exchange and can lead to hypoxemia. ${ }^{13}$

\section{LUNG COMPLIANCE}

The chest tube and standard bedside pleural drainage system also provide insights into lung compliance.

A rationale for the empirical routine of $-20 \mathrm{~cm} \mathrm{H}_{2} \mathrm{O}$ of suction for patients-approximately $10 \mathrm{~cm} \mathrm{H}_{2} \mathrm{O}$ below normal pleural pressures-provides insurance that average pressures will remain negative despite transient increases in pleural pressure. Depending on lung compliance, the increased suction (more negative pleural pressures) might also facilitate lung expansion. The relative utility of increasing pleural suction to expand the lung and eliminate a pleural air space is determined by the lung's compliance (Figure 4, A). In the spontaneously ventilating patient with an esophageal balloon, lung compliance is typically measured during interrupted expiration and calculated as the ratio of lung volume change to transpulmonary pressure change. If the lung is on the compliant (linear) portion of the curve (Figure 4, $A, \mathrm{~V}_{\mathrm{n}}$ ), then parenchymal expansion might occur with modest changes in chest tube suction. In contrast, an overexpanded and relatively noncompliant lung (eg, postresection lung; Figure $4, A, \mathrm{~V}_{\mathrm{hi}}$ ) is unlikely to increase its volume in response to increased pleural suction.

Excessive suction also has disadvantages. Rather than increasing overall lung expansion, increased suction might simply distort the local pleural surface at the end of the thoracostomy tube, ${ }^{14}$ thereby sucking the surface of the lung against the chest tube resulting in the functional occlusion (exclusion) of the chest tube. In patients with a large air 

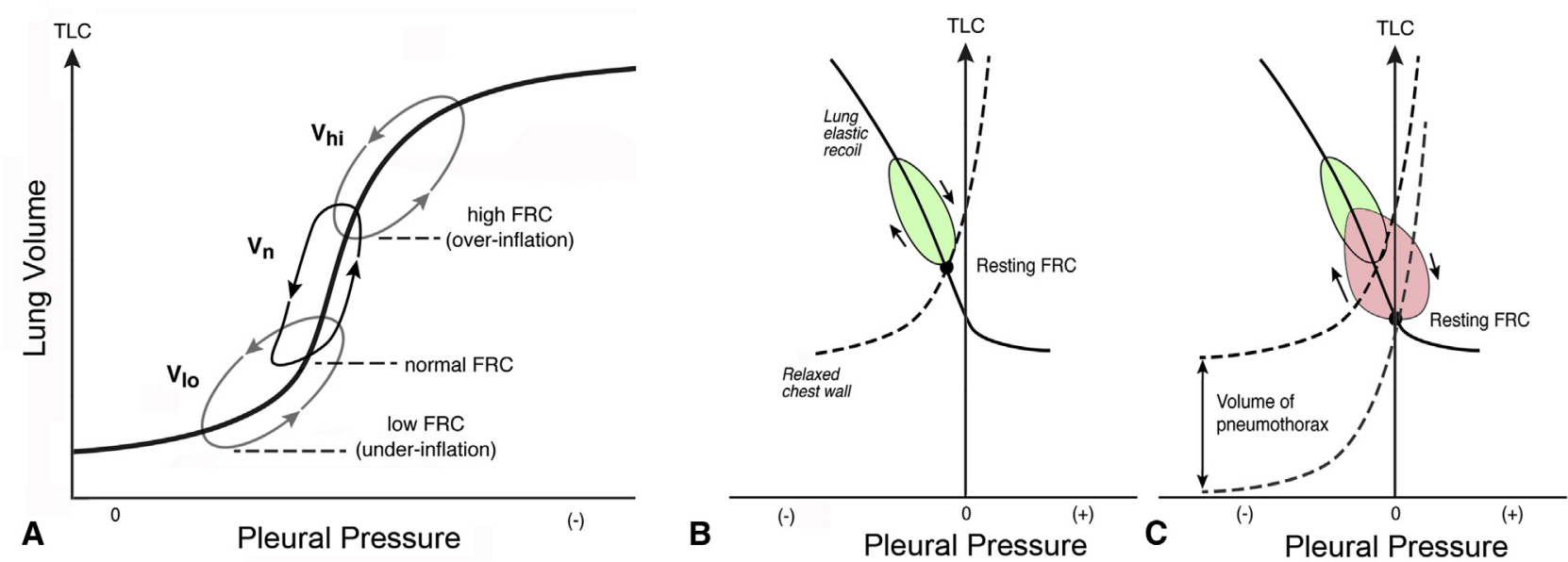

FIGURE 4. A, Pressure-volume (PV) curve of the lung. At low lung volumes (analogous to a deflated balloon; $V_{\mathrm{lo}}$ ), the lung is noncompliant and significant changes in transpulmonary pressures are required to increase lung volumes. Atelectasis is often associated with this range of lung volumes. In the compliant portion of the curve $\left(V_{\mathrm{n}}\right)$, changes in transpulmonary pressure result in efficient changes in lung volume. Functional residual capacity $(F R C)$ defines the lower end of this portion of the curve. At high lung volumes (analogous to an overinflated balloon; $V_{\mathrm{hi}}$ ), changes in transpulmonary pressures result in less efficient changes in lung volumes. The maximum lung volume defines total lung capacity $(T L C)$. B and $\mathrm{C}$, Campbell diagram of pleural pressure versus lung volume ${ }^{16}$ illustrating the changes in breathing associated with a pneumothorax. The lung's elastic PV curve (solid line) and the PV curve of the relaxed chest wall (dashed line) are shown. ${ }^{16} \mathrm{~B}$, In the spontaneous breathing patient, the resting FRC is at the intersection of the lung and relaxed chest wall curves. The loop shows the pleural pressure-volume trace during a spontaneous breath (dark gray). C, With a pneumothorax, the relaxed chest wall PV curve shifts downward, reflecting the volume of the pneumothorax. After pneumothorax, the spontaneous breathing loop (light gray) reflects the greater change in pleural pressure needed for a given change in lung volume (due to increased pulmonary resistance and elastance at a lower FRC).

leak, it is common practice to place at least 2 tubes, positioned at the extremes of the chest (eg, apical and basilar), to facilitate lung expansion and avoid parenchymal exclusion of the tubes.

\section{Noncompliant Lung}

When lung volumes are within the normal range, the respiratory system is operating in the most compliant portion of the pressure-volume (PV) curve (Figure 4, A, $\mathrm{V}_{\mathrm{n}}$ ). In the spontaneously breathing patient, the decrease of lung volumes to the nonlinear, and noncompliant range of the PV curve means that large changes in transpulmonary pressure are required to maintain baseline tidal ventilation (Figure $4, A, \mathrm{~V}_{\mathrm{lo}}$ ). In the beside pleural drainage system, or simply in the chest tube fluid, these amplified changes in pleural pressure are reflected by large tidal swings in the column of fluid. In the mechanically ventilated intensive care unit patient, the noncompliant lung requires similarly high transpulmonary pressures, but without significant changes in pleural pressures, the intensive care unit patient will require increased airway pressures to maintain lung volumes.

In patients with a "big swing" in chest tube pressure, the clinical issue is to identify the reason for lung volume loss. In postsurgical patients, the progressive loss in lung volume might be caused by inactivity-induced atelectasis, compliance changes because of overhydration ("wet" lungs), or airway obstruction from mucous plugging. In many cases, attention to the intake/output fluid balance as well as aggressive mobilization of the patient can improve airway clearance and recruit lung volumes.

A noncompliant lung might also be observed at the other extreme of the PV curve; that is, at higher lung volumes (Figure $4, A, \mathrm{~V}_{\mathrm{hi}}$ ). A common reason for noncompliance is surgical resection of the lung. ${ }^{15}$ The remaining lung might be overinflated and operating on the upper portions of the PV curve. In this case, increasing the suction on the chest tube is unlikely to increase the volume of the hyperexpanded lung.

\section{Chest Wall and Lung Interactions}

The work of breathing, the work performed by respiratory muscles, can be graphically represented on the Campbell diagram; that is, a plot of pleural pressure and lung volume (Figure $4, B$ ). ${ }^{16}$ The solid line shows pleural pressures developed by the chest wall during a slow active inspiration. The dashed line corresponds to the elastic pressure of the completely relaxed chest wall. The pressure difference between these active and relaxed states is the result of muscle action.

The Campbell diagram usefully illustrates the effects of a pneumothorax. A pneumothorax changes the relative positions of the PV curves of the elastic characteristics of the chest wall and lung. Because of the pneumothorax, the 
lung volume is less than normal and the chest wall volume is greater. As a result, the PV curve of the relaxed chest wall is displaced downward commensurate with the size of the pneumothorax (Figure 4, C). Inspiration from this lower resting functional residual capacity, where resistance and elastance are increased, requires an amplified swing in pleural pressure and increased work of respiratory muscles.

\section{PLEURAL SPACE}

The hydrostatic pressure gradient from the top to the bottom of the lung is a consequence of gravity and posture. ${ }^{14} \mathrm{In}$ the upright patient, the effect of gravity is reflected in the apical migration of pleural air. With a large accumulation of air, the hydrostatic pressure gradient is eliminated; the pleural pressures will be uniform throughout the air bubble in the pleural air space. The effects of gravity can be important, however, in maintaining tube position and avoiding tube exclusion.

The benefits of an apical tube include not only the evacuation of apical air, but the displacement of the lung toward the apex of the chest. A lesson learned from tuberculosis surgery during the 1950s and 1960s was the importance of visceral-parietal pleural apposition in healing air leaks. ${ }^{17}$ Although the mechanism of the pleural healing process is unknown, a potential explanation for this observation is the sliding movement of the pleura. During normal breathing the apex of the lung is relatively immobile and does not slide against the chest wall; therefore, the apex of the lungs provides a static surface area for apposition of the pleural surfaces. In contrast, the base of the chest is characterized by respiratory sliding movements between the visceral and parietal pleural surfaces. ${ }^{18}$

There are surgical techniques for facilitating apical displacement and pleural apposition. After an upper lobe resection in a relatively noncompliant lung, mobilizing the inferior pulmonary ligament might help facilitate displacement of the lower lobe cephalad. ${ }^{19}$ Of historic interest, surgical techniques designed to eliminate pleural space and facilitate pleural apposition included thoracoplasty, ${ }^{20}$ pleural partitioning, ${ }^{21}$ diaphragmatic reimplantation, ${ }^{20}$ and pneumoperitoneum. ${ }^{22}$ A practice designed to elevate the diaphragm was phrenic nerve "crush" injury; however, this practice is increasingly uncommon because of the acknowledged role of cyclic ventilation in normal breathing as well as lung repair. ${ }^{23}$

In some cases, pleural adhesions will limit the apical displacement of the remaining lung. If possible, the pleural adhesions should be fully mobilized to permit the cephalad displacement of the lung. In unusual situations, in which the lung cannot be mobilized, the apical parietal pleura can be dissected from the apical chest wall creating a "pleural tent." ${ }^{24}$ The pleural tent converts the intrapleural space into an extrapleural space; more importantly, it facilitates pleural apposition and the healing of air leaks. Interestingly, the extrapleural space appears to be resistant to infection and gradually resorbs over several months.

The functional role of the pleural space in human ventilation remains unclear, but a potentially important function is the coupling of diaphragm movement to alveolar ventilation in remote regions of the lung. Pleurodesis, especially at the bases of the lungs, limits normal diaphragm sliding movements and might influence ventilation in the lung. ${ }^{25}$ Although these effects are not significant in most patients, there are special cases particularly vulnerable to pleurodesis. For example, patients with bilateral pleurodeses and only 1 functioning diaphragm have a striking incidence of hypercarbic respiratory failure.

\section{Conflict of Interest Statement}

Authors have nothing to disclose with regard to commercial support.

\section{References}

1. Mead J, Gaensler EA. Esophageal and pleural pressures in man, upright and supine. J Appl Physiol. 1959;14:81-3.

2. Loring SH, Topulos GP, Hubmayr RD. Transpulmonary pressure: the importance of precise definitions and limiting assumptions. Am J Respir Crit Care Med. 2016;194:1452-7.

3. Akoumianaki E, Maggiore SM, Valenza F, Bellani G, Jubran A, Loring SH, et al. The application of esophageal pressure measurement in patients with respiratory failure. Am J Respir Crit Care Med. 2014;189:520-31.

4. Loring SH, Malhotra A. Driving pressure and respiratory mechanics in ARDS. $N$ Engl J Med. 2015;372:776-7.

5. Liang SY, Ivanovic J, Gilbert S, Maziak DE, Shamji FM, Sundaresan RS, et al. Quantifying the incidence and impact of postoperative prolonged alveolar air leak after pulmonary resection. J Thorac Cardiovasc Surg. 2013;145:948-54.

6. Cerfolio RJ, Bass CS, Pask AH, Katholi CR. Predictors and treatment of persistent air leaks. Ann Thorac Surg. 2002;73:1727-30.

7. Villena V, Lopez-Encuentra A, Pozo F, De-Pablo A, Martin-Escribano P. Measurement of pleural pressure during therapeutic thoracentesis. Am J Respir Crit Care Med. 2000;162:1534-8.

8. Gocyk W, Kuzdzal J, Wlodarczyk J, Grochowski Z, Gil T, Warmus J, et al. Comparison of suction versus nonsuction drainage after lung resections: a prospective randomized trial. Ann Thorac Surg. 2016;102:1119-24.

9. Brunelli A, Beretta E, Cassivi SD, Cerfolio RJ, Detterbeck F, Kiefer T, et al. Consensus definitions to promote an evidence-based approach to management of the pleural space. A collaborative proposal by ESTS, AATS, STS, and GTSC. Eur J Cardiothorac Surg. 2011;40:291-7.

10. Rutherford RB, Hurt HH, Brickman RD, Tubb JM. Pathophysiology of progressive tension pneumothorax. J Trauma. 1968;8:212-27.

11. Filoche M, Felici M, Sapoval B. Renormalized random walk study of oxygen absorption in the human lung. Physica A. 2005;357:27-35.

12. Bishop MJ, Benson MS, Pierson DJ. Carbon-dioxide excretion via bronchopleural fistulas in adult respiratory-distress syndrome. Chest. 1987;91:400-2.

13. Downs JB, Chapman RL. Treatment of bronchopleural fistula during continuous positive pressure ventilation. Chest. 1976;69:363-6.

14. Laifook SJ, Rodarte JR. Pleural pressure distribution and its relationship to lung volume and interstitial pressure. J Appl Physiol. 1991;70:967-78.

15. Salito C, Bovio D, Orsetti G, Salati M, Brunelli A, Aliverti A, et al. Effect of lung resection on pleuro-pulmonary mechanics and fluid balance. Respir Physiol Neurobiol. 2016;221:35-40.

16. Campbell EJ. The Respiratory Muscles and the Mechanics of Breathing. London: Lloyd-Luke; 1958.

17. Shields TW, Lees WM, Fox RT, Salazar G. Persistent pleural air space following resection for pulmonary tuberculosis. J Thorac Cardiovasc Surg. 1959;38: 523-36.

18. Kim JH, Butler JP, Loring SH. Influence of the softness of the parietal pleura on respiratory sliding mechanisms. Respir Physiol Neurobiol. 2011;177:114-9. 
19. Khanbhai M, Dunning J, Yap KH, Rammohan KS. Dissection of the pulmonary ligament during upper lobectomy: is it necessary? Interact Cardiovasc Thorac Surg. 2013;17:403-6.

20. Bjork VO. The surgical treatment of lower lobe tuberculosis. J Thorac Surg. 1956;31:655-71.

21. Brewer LA, Bai AF, Jones WM. The development of the pleural partition to prevent overexpansion of the lung following partial pulmonary resection - an experimental study with clinical application. J Thorac Surg. 1956;31:165-82.

22. Loring SH, Behazin N, Novero A, Novack V, Jones SB, O'Donnell CR, et al. Respiratory mechanical effects of surgical pneumoperitoneum in humans. $J$ Appl Physiol. 2014;117:1074-9.
23. Loring SH, Mead J, Griscom NT. Dependence of diaphragmatic length on lung volume and thoracoabdominal configuration. J Appl Physiol. 1985;59:1961-70.

24. Rainer WG, Newby JP. Prevention of residual space problems after pulmonary resection. Am J Surg. 1967;114:744-7.

25. Loring SH, Kurachek SC, Wohl ME. Diaphragmatic excursion after pleural sclerosis. Chest. 1989;95:374-8.

Key Words: pleural injury, pneumothorax, transpulmonary pressure 